S. J. TANTRY, V. V. S. BABU* (BANGALORE UNIVERSITY, INDIA)

9-Fluorenylmethyl Chloroformate (Fmoc-Cl) as a Useful Reagent for the Synthesis of Pentafluorophenyl, 2,4,5-Trichlorophenyl, Pentachlorophenyl, $p$-Nitrophenyl, $o$-Nitrophenyl and Succinimidyl Esters of $\mathrm{N}^{\alpha}$-Urethane Protected Amino Acids

Lett. Pept. Sci. 2003, 10, 655-662.

\title{
Synthesis of Reactive Amino Acid Esters by Using 9-Fluorenylmethyl Chloroformate
}

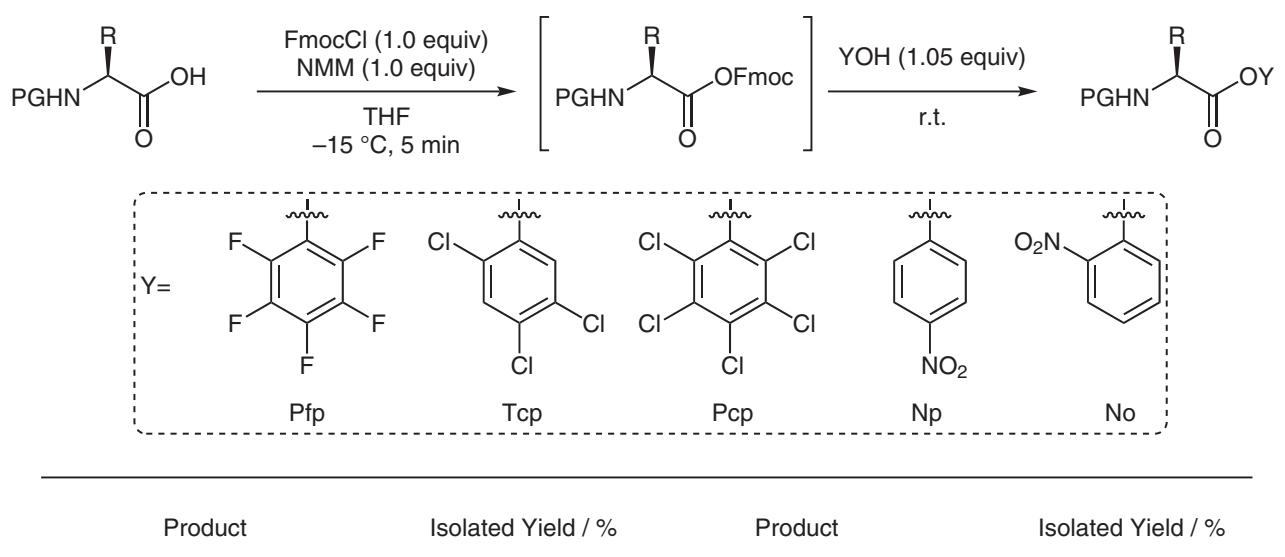

Fmoc-Phe-OPfp
Fmoc-D-Phe-OPfp
Fmoc-Ser(t-Bu)-OPfp
Fmoc-Asp(t-Bu)-OPfp
Fmoc-Lys(Cbz)-OPfp
Fmoc-Cys(Acm)-OPfp
Fmoc-His(Trt)-OPfp
Fmoc-Asn-OPfp
Fmoc-Gln-OPfp
Cbz-Leu-OPfp
Cbz-Ser-OPfp

95
92
85
90
95
80
72
81
78
90
80

$\begin{array}{lc}\text { Cbz-Asp(t-Bu)-OPfp } & 90 \\ \text { Fmoc-Phe-OTcp } & 90 \\ \text { Fmoc-Cys(Bzl)-OTcp } & 95 \\ \text { Boc-Leu-OPcp } & 90 \\ \text { Cbz-Glu(t-Bu)-OTcp } & 80 \\ \text { Cbz-Cys(Bzl)-OPcp } & 90 \\ \text { Fmoc-Ala-ONp } & 90 \\ \text { Fmoc-Val-ONo } & 84 \\ \text { Fmoc-Lys(Boc)-ONp } & 80 \\ \text { Cbz-Gly-ONp } & 93 \\ \text { Cbz-Cys(Bzl)-ONp } & 90\end{array}$

Significance: Reactive amino acid phenyl esters have been used in dipeptide synthesis. However, the preparation of these esters is challenging due to racemization during the formation of the esters. In 2003, Tantry and Babu reported a method in which $\mathrm{FmocCl}$ is used to prepare reactive amino acid esters.

\section{Category}

Peptide Chemistry

\section{Key words}

fluorenylmethyl chloroformate

phenols

amino acid esters

racemization

mixed anhydrides
Comment: With the developed method, various reactive amino acid phenyl esters can be synthesized. The yields of the reactions are excellent, and no racemization is detected. 